\title{
Neural Network Load Forecasting Model Based on BP
}

\author{
Xi Gao* \\ School of the North China Electricity Power University, Baoding 071000, China; \\ 454413105@qq.com
}

\begin{abstract}
Short-term load forecasting is the basis of power system operation and analysis, which is of great significance for unit commitment, economic load dispatching, safety checking and so on. As a result, improving the accuracy of load prediction is an important way to ensure the scientific decision-making of power system optimization. In this paper, based on the annual load data in two regions, we predict the discrete data by the BP neural network load forecasting model, to get the load forecast data for a given period of time in the future. In the end, the advantages and disadvantages of the two regions are evaluated, and we can get the better prediction model.
\end{abstract}

Keywords: neural network model, nonlinear regression, Short-term load forecasting

\section{Introduction}

Due to the multiply uncertain and complex factors such as temperature, climate, holiday and so on, time series of the power load is of highly nonlinear, time varying and uncertainty. And time series has different sensitive degree to input parameters. So it is difficult to use the conventional prediction methods (such as the regression analysis methods) to predict the trends of power load accurately. As a result, power load forecast need to introduce lot of new theories. Artificial neural network is composed of many simple, parallel neurons, which is trained in a given sample. By extracting the nonlinear mapping relation, we can solve the problem of multivariate nonlinear regression effectively, which is one of the most widely used methods in the field of power load forecasting.

In 1986, Rumelhart and McClelland put forward a neural network basing on error back propagation training algorithm, which was known as BP neural network. Multilayer BP neural network can approximate any nonlinear continuous map in theory so it is very suitable for modeling the system of nonlinear regression. In addition, the determination of the number of layers and the number of neurons in hidden layer not only help reduce the operation cost, but also improve the operation speed. And selecting the appropriate neurons has a great effect on the accuracy of the prediction, too.

\section{Double hidden layer BP neural network}

\section{1 setting up a BP neural network}

This model establishes a Multiple-Input and Multiple-Output BP neural network model with double hidden layer. The network consists of 97 input layer neurons and 
96 output layer neurons. Compared with the conventional single hidden layer BP neural network, although in theory the single hidden layer neural network can approximate any nonlinear continuous mapping, its convergence speed is slower and shows the probability of local convergence. By contrast, the convergence speed of double hidden layer BP neural network has obviously improved (if other conditions are the same) ${ }^{[1]}$. So we choose the feedforward neural network with double hidden layers to finish this model.

\section{2 determining the number of hidden layer neurons}

According to the reference ${ }^{[2]}$, by referring to the following three formulas, we can determine the best number of hidden layer units.

$$
\begin{aligned}
& \begin{array}{l}
\text { Formula } 1 \quad n_{1}=\log _{2} n
\end{array} \\
& \begin{array}{l}
\text { Formula } 2 \quad n_{1}=\sqrt{n+m}+a
\end{array} \\
& \text { Formula } 3 \\
& \sum_{i=0}^{n} C_{n_{1}}^{i}>k
\end{aligned}
$$

In these formulas, $\boldsymbol{n}_{1}$ is the number of hidden layer units. $\boldsymbol{n}$ is the number of input units. $\boldsymbol{m}$ is the number of output units. $a$ is a constant between 1 and $10 . k$ is a sample number. We choose the three formulas for best hidden layer selection, respectively.

\subsection{Training principles}

By the training principle, according to the known load data in a week, we can predict the power load in the next seven days. Assume that there are 96 sets of data per day (produce a set of data every 15 minutes). So, in the training data, the input data of the day is connected with the output data of the day in next week.

\subsection{The principles for data selection}

After the establishment of BP neural network, training data are needed to train the network. And the selection of data requires given rules as follows:

(1)Considering the closest historical data

Because of the short time, the trend of power load tends to be smooth and continuous. Therefore, the change rule of adjacent time points in a short time should be similar and there is little distortion.

(2)Considering cyclical impact

According to the data analysis, there is a quasi periodic variation of the load. The power load of Sunday per week decreased significantly over other 6 days in this week. So when we train data, in addition to input power load data every day (96 sets of data) to produce each sample of input layer, we need to input periodic mark, which represents the day of a week. 


\section{Numerical calculation}

\subsection{Neuron in hidden layer}

According to 2.2, we calculate the number of hidden layer units by using 3 formulas, with $6,[14,23],[4, \infty]$ respectively. We trained the hidden layers within the range, to get the result whose mean square error is minimum and the computation time is short. When the number of neurons in the hidden layer is 4 and 6 , respectively, the square error of training data is minimal, which are shown as follows:

\begin{tabular}{ccc} 
Table 1 & the number of neurons in the hidden layer \\
\hline & the hidden layer 1 & the hidden layer 2 \\
\hline the number of neurons & 4 & 6 \\
\hline
\end{tabular}

\subsection{Selecting the training data}

According to the principles for data selection, we choose data in four straight weeks before January 11, 2015 to calculate the BP neural network. The number of samples in this period is $28 \times 96$.

According to the periodicity, based on the 96 predicted values of every sample, we add the sequential number of the day in a week (1 to 7). So the number of input layer neurons is 97 and the number of output layer neurons is 96 . And results are as follows:

Table 2 Input and output sample correspondence

\begin{tabular}{cc}
\hline Input layer data & Output layer data \\
\hline (load in 20141214)、Sunday(7) & load in 20141221 \\
(load in 20141215)、Monday(1) & load in 20141222 \\
(load in 20141216)、Tuesday(2) & load in 20141223 \\
$\vdots$ & $\vdots$ \\
(load in 20150103)、Saturday(6) & load in 20150110 \\
\hline
\end{tabular}

\subsection{Training parameters setting and prediction results}

The training parameters are as follows:

Table 3 Settings

\begin{tabular}{cc}
\hline Parameters & Values \\
\hline $\begin{array}{c}\text { Maximum training time } \\
\text { Number of neurons in hidden } \\
\text { layer }\end{array}$ & 500 \\
$\begin{array}{c}\text { the training speed of the } \\
\text { network } \\
\text { the target error }\end{array}$ & 0.1 \\
Add momentum factor or not & 0.001 \\
Not
\end{tabular}

According to the parameter setting principle above, we do the neural network training simulation. And the network structures are as follows: 


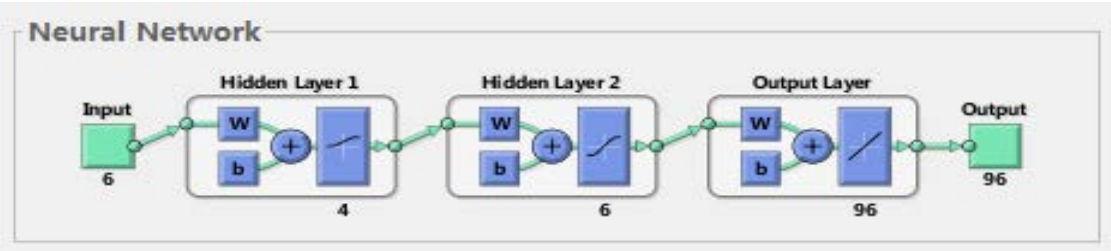

Fig.1 BP neural network structure

Using the trained neural network, by the training principle, we can get the prediction results as follows:

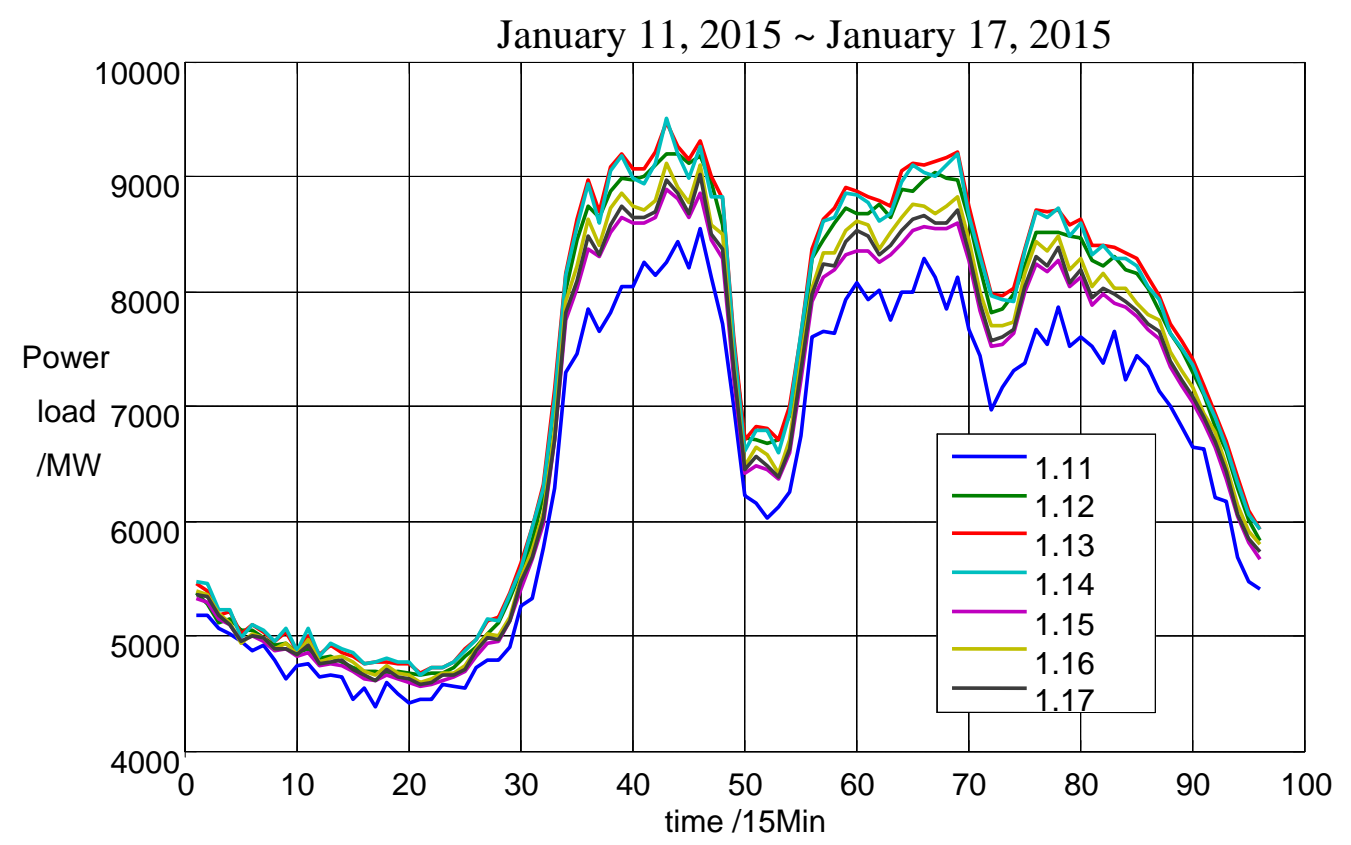

Fig. 2 prediction results of area 1

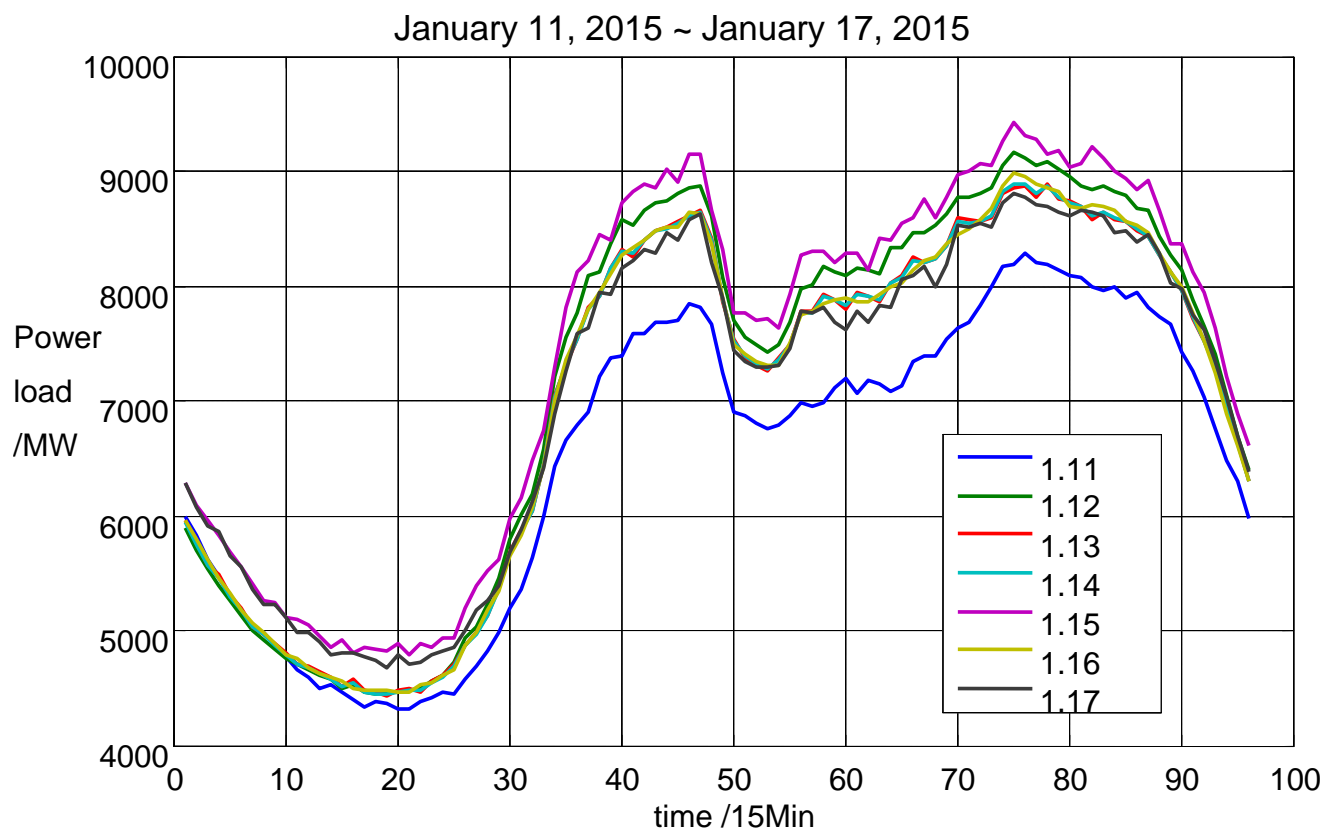

Fig. 2 prediction results of area 2 


\section{Model conclusion}

Based on the historical load data, we can predict the short-term load of power system, but the accuracy of prediction is affected by the change of load, the forecast method and meteorological factors.

Through our model, we find that the change of the load in a region can meet the following characteristics:

(1)Regularity. The main influence factor is the quasi periodic variation trend in a week. The larger the load cycle is, the stronger the regularity of the load will be.

(2)Randomness. The main factor is the base of the load. The greater the load base is, the less the degree of uncertainty is.

(3)Stability. It is mainly reflected in the bearing capacity of the holiday.

The main meteorological factor to improve the forecast accuracy is the daily maximum temperature, and the daily average temperature and daily minimum temperature have a significant impact on the prediction accuracy.

Because of the nonlinear load in power system, the general regression fitting method can only approximate the load trend, and accuracy still needs to be improved. At the same time, BP neural network algorithm can predict multiple nonlinear regression problems well.

\section{References}

[1]Yuhua Shen, Zhaoxia Wang. Determination of the number of hidden layer units in BP neural network[J]. Journal of Tianjin University of Technology, 2008,24(5):13-15. [2]Xiaoling Shen. Study on short term load forecasting of power system[D]. Tianjin University,2009. 